\title{
An Overview of the Most Common Effective Antioxidants on Hemodialysis
}

\author{
Aliasghar Manouchehri ${ }^{1}$, Pardis Ghanadi ${ }^{2}$, Mostafa Cheraghi ${ }^{3}$, Fatemeh MehrabiRad $^{4}$, Fakher \\ Biglaryan $^{5}$, Mehrnoosh Sedighi $^{3, *}$, Leila Mahmoodnia ${ }^{6}$ \\ ${ }^{1}$ Department of Internal Medicine, Shahid Beheshti Hospital, Babol University of Medical Sciences, Babol, Iran \\ ${ }^{2}$ Lorestan University of Medical Sciences, Khorramabad, Iran \\ ${ }^{3}$ Razi Herbal Medicines Research Center \& Cardiovascular Research Center, Shahid Rahimi Hospital, Lorestan University of Medical \\ Sciences, Khoramabad, Iran \\ ${ }^{4}$ Student Research Committee, Faculty of Medicine, Lorestan University of Medical Sciences, Khorramabad, Iran \\ ${ }^{5}$ Master of Clinical Psychology and Head of the Health Department of Chalous Police, NAJA, Mazandaran, Iran \\ ${ }^{6}$ Department of Internal Medicine, School of Medicine, Shahrekord University of Medical Sciences, Shahrekord, Iran
}

Received December 27, 2020; Revised February 22, 2021; Accepted March 1, 2021

\section{Cite This Paper in the following Citation Styles}

(a): [1] Aliasghar Manouchehri, Pardis Ghanadi, Mostafa Cheraghi, Fatemeh MehrabiRad, Fakher Biglaryan, Mehrnoosh Sedighi, Leila Mahmoodnia , "An Overview of the Most Common Effective Antioxidants on Hemodialysis," Advances in Pharmacology and Pharmacy, Vol. 9, No. 1, pp. 9 - 15, 2021. DOI: 10.13189/app.2021.090102.

(b): Aliasghar Manouchehri, Pardis Ghanadi, Mostafa Cheraghi, Fatemeh MehrabiRad, Fakher Biglaryan, Mehrnoosh Sedighi, Leila Mahmoodnia (2021). An Overview of the Most Common Effective Antioxidants on Hemodialysis. Advances in Pharmacology and Pharmacy, 9(1), 9 - 15. DOI: 10.13189/app.2021.090102.

Copyright $\bigcirc 2021$ by authors, all rights reserved. Authors agree that this article remains permanently open access under the terms of the Creative Commons Attribution License 4.0 International License

\begin{abstract}
Dialysis itself increases oxidative stress. The causes of oxidative stress in patients are not yet known, but uremic toxins, dialyzer interactions, and dialysis fluid contamination have been suggested as three major causes of the stress. Peripheral blood cell-dialysis membrane interaction is another hypothesis that has been proposed for increase in the production of reactive oxidant species. Antioxidants can improve or prevent disease by affecting the involved factors. This article aims to introduce antioxidants that affect injuries due to hemodialysis to familiarize the readers with the disease and prevent and treat the disease using antioxidants. For this purpose, the important effects of medicinal plants with antioxidant properties on the levels of production of reactive oxygen species (ROS), nitrogen (NO), peroxynitric acid, protein oxidation, lipid peroxidation and reduced malondialdehyde (MDA) production and apoptosis in dialysis were investigated and reported. To conduct this review, relevant articles retrieved from Pubmed, ISI and Scopus databases using search terms antioxidant, reactive oxygen species (ROS), dialysis, and kidney, and indexed from 1991 to 2019 were used. Herbal active ingredients such as phenolic compounds, flavonoids and antioxidant compounds can contribute to reducing. It also prevents hemodialysis incidence and its
\end{abstract}

adverse effects by preventing increase of free radicals, decrease of membrane lipid peroxidation, and increase of antioxidant enzymes catalase (CAT), superoxide dismutase (SOD), and glutathione peroxidase (GPX). Free radicals affect fat, protein, DNA and cell membranes, including membrane carbohydrates, causing membrane damage and cell disintegration. In terms of clinical applications, antioxidant products can be used to treat oxidative stress related to dialysis and are practical drugs. In addition, small proteins such as immunoglobulin $\mathrm{G}$ and complements attach to the membrane of the dialysis machine which activate granulocytes and produce free radicals. Antioxidants with prevent of kidney damage and progression of the disease by affecting the factors effective on hemodialysis.

Keywords Dialysis, Antioxidant, Free radical, Treatment

\section{Introduction}

Free radicals are molecules that are chemically highly reactive. The production of free radicals is a natural 
process of the body's metabolic reaction. The molecules are involved in diseases such as diabetes, cancer, rheumatoid arthritis, and inflammatory, infectious, kidney and cardiovascular diseases. Free radicals are eliminated from the body by combining with antioxidants [1]. Patients with chronic kidney disease and patients who are routinely dialysed are more likely to develop premature cardiovascular disease. Overproduction of free radicals leads to conditions called oxidative stress. Oxidative stress is probably one of the causes of vascular lesions $[1,2]$. Free radicals affect the cell membrane and cause lipid peroxidation, destruction of molecules and cell (endothelial cell and erythrocyte) structure [2]. There is ample evidence that chronic kidney failure patients are constantly exposed to oxidative stress. Dialysis itself increases oxidative stress. The causes of oxidative stress in diabetic patients are not yet known, but uremic toxins, dialyser interactions, and dialysis fluid contamination have been suggested as three major causes of this stress [2-4]. Peripheral blood cell-dialysis membrane interaction is another hypothesis that has been proposed for increased production of reactive oxidant species. Small proteins, such as immunoglobulin $G$ and complement, may also bind to the dialysis membrane, activating granulocytes and producing free radicals. An active antioxidant system is therefore required. Evidence indicates that the antioxidant system of these patients is defective. Maladaptation of dialysis membranes with the biological environment of the blood plays a major role in increasing the production of oxygen free radicals and the risk of sclerotic lesion $[3,4]$. Uremic syndrome also plays a part in the production of free radicals and damage to the cell membrane [5]. The prevalence and mortality from cardiovascular diseases is high in patients with kidney failure. Multiple factors such as dyslipidemia, hypertension, diabetes, and elevated fibrinogen and homocysteine levels can affect the progression of the disease. The direct role of inflammation and involvement of reactive oxygen species (ROS) has been mediated by activation of macrophages in the arterial wall [6]. Increase in oxidative stress of lipids and proteins, formation of fatty streaks and atherosclerosis, and prevalence of heart disease are also extremely high in hemodialysis patients [6, 7]. Despite the conducted research on increase of oxidative stress in these patients, the results in patients treated with peritoneal dialysis and hemodialysis are inconsistent. Serum malondialdehyde (MDA) levels in these patients are important as an indicator of lipid peroxidation. Antioxidant activities of catalase (CAT), glutathione peroxidase (GPX), and superoxide dismutase (SOD) are high in glomeruli and in equilibrium with oxidative stress [8]. Diet therapy may help patients by providing counseling and training techniques. Adherence to serum phosphate, potassium, and blood urea nitrogen levels indicates the rate of patient adherence to dietary and medication regimens. While weight gain between the two dialysis sessions indicates the patient's adherence to fluid regimens $[9,10]$. Maintaining potassium balance is important more critical in patients undergoing dialysis. This can be made possible by imposing restrictions on dietary and medication regimens that are more difficult for patients to follow simultaneously [11]. The amount of protein intake is important depending on the economic situation [12]. Results have shown that $56 \%$ of patients do not adhere to fluid restriction. Schneider et al. reported lack of adherence to fluid regimens in patients with renal failure as being approximately $33 \%$ [13]. In other studies, this rate has been reported to range between $25 \%$ and $80 \%$ [14]. The detrimental effects of serum phosphate levels become apparent to patients over time, yet they are not as harmful as the effects of elevated potassium level. High potassium has a detrimental effect on the kidneys, causing chronic kidney disease, leading to anemia, bone weakness, nerve damage, and eventually kidney failure, heart disease, and premature death $[13,14]$. The quality of hemodialysis is effective on people's longevity and can also reduce the risk of cardiovascular disease. This is due to the modulation of the metabolic disorders caused by uremic status in these patients [15]. This article aims to introduce antioxidants that affect injuries due to hemodialysis to familiarize the readers with the disease and prevent and treat the disease using antioxidants.

\section{Method for Review}

To conduct this review, relevant articles retrieved from Pubmed, ISI and Scopus databases using search terms antioxidant, reactive oxygen species (ROS), dialysis, and kidney, and indexed from 1991 to 2019 were used (Nearly three decades).All articles were searched and downloaded based on specific words and articles and texts were used to review the article.

\section{Results}

Plant active ingredients such as phenolic compounds, flavonoids and antioxidant compounds can contribute to reducing and preventing hemodialysis incidence and its adverse effects by preventing increase of free radicals, decrease of membrane lipid peroxidation, and increase of antioxidant enzymes catalase (CAT), superoxide dismutase, From this category we can name such as Klatho, Taurine, Adiponectin, N-acetylcysteine, Folic acid and B12, L-carnitine, Vitamins C, Nicotinamide, Quercetin, Selenium and Thea-lipoic acid. Table 1 summarizes the above compounds with explanations of their effects. 
Table1. Compounds affecting on hemodialysis

\begin{tabular}{|l|l|}
\hline Klotho & Active vitamin D is a positive factor for klotho gene expression \\
\hline Taurine & Taurine scavenges ROS and enhances renal function \\
\hline Adiponectin & $\begin{array}{l}\text { adiponectin level, resistance, MCP-1, and adipsin levels were significantly elevated in dialysis patients, leading to } \\
\text { increased renal clearance. }\end{array}$ \\
\hline N-acetylcysteine & NAC effectively induces kidney function in patients with chronic hemodialysis. \\
\hline $\begin{array}{l}\text { Folic acid and } \\
\text { B12 }\end{array}$ & $\begin{array}{l}\text { Decrease of its scavenging leads to risk factors for thrombosis and coronary artery disease, pulse pressure, arterial } \\
\text { stiffness, and platelet aggregation }\end{array}$ \\
\hline L-carnitine & $\begin{array}{l}\text { L-carnitine reduces the production of Acetyl-CoA, thereby reducing the production of free radicals, helping to repair } \\
\text { oxidative stress to the phospholipid membrane, and reducing ischemia induced by apoptosis. }\end{array}$ \\
\hline Vitamins C, E & $\begin{array}{l}\text { The imbalance between the anti-peroxide system and antioxidant system is comparably greater in patients with kidney } \\
\text { failure. }\end{array}$ \\
\hline Nicotinamide & Nicotinamide may be considered as a treatment of choice for reducing the endotoxin load in hemodialysis patients. \\
\hline Quercetin & $\begin{array}{l}\text { Treatment with quercetin in hemodialysis patients improved renal function, reduced oxidative stress, and decreased } \\
\text { serum fibroblast growth. }\end{array}$ \\
\hline Selenium & $\begin{array}{l}\text { Selenium is an essential trace element that functions as a cofactor for the reduction of antioxidant enzymes such as } \\
\text { glutathione peroxidase. }\end{array}$ \\
\hline Thea-lipoic acid & \begin{tabular}{l} 
Thea-lipoic acid decreased plasmaasymmetric dimethylarginine \\
\hline
\end{tabular}
\end{tabular}

Klotho: The positive correlation between klotho and 8-OHDG in men can on the hemodialysis group may be a compensatory mechanism in which klotho is increased to compare with oxidative stress [16]. Serum klotho concentrations are higher in hemodialysis patients than in kidney transplant patients, which may be due to low serum inactive vitamin D concentration, or perhaps due to immunosuppressive drugs in kidney transplant patients. Higher parathyroid hormone levels and lower levels of inactive vitamin $\mathrm{D}$ in hemodialysis patients can explain comparably higher klotho levels in these patients. Active vitamin $\mathrm{D}$ is a positive factor for klotho gene expression, and therefore parathyroid can increase klotho through active vitamin D [17]. The inactive form reduces klotho [18].

Taurine: Taurine contributes to a number of various physiological and biological processes in the kidney, often reflected in urine excretion, and is important for kidney and body homeostasis. As an important antioxidant, taurine plays an important role in renal and vascular endothelial blood flow. In glomerulus, inflammatory mechanisms of cytokines cause leukocyte migration, T-cell activation, fibrosis, sclerosis, and ulceration. Taurine scavenges ROS and enhances renal function. Taurine plays a part in cell volume and cell osmolality, especially the renal medulla and final urine volume. Taurine is a potent antioxidant that plays a role in phagocytosis and inflammation reduction and serves a protective function against hemodialysis, ischemia and various kidney diseases [19].

Adiponectin: Adiponectin is the most abundant peptide secreted by adipocytes, whose reduction plays a central role in obesity-related diseases, including insulin resistance/type 2 diabetes and cardiovascular disease. Adiponectin levels are high in hemodialysis patients, Okuno et al. found that increased adiponectin levels were associated with hemodialysis. In a study on 368 patients, Liu et al. reported that adiponectin level, resistance,
MCP-1, and adipsin levels were significantly elevated in dialysis patients, leading to increased renal clearance. Serum adiponectin levels were inversely correlated with GFR, BMI, glucose, LDL, and platelet levels, whereas a direct correlation was observed with creatinine level. Notably, adiponectin is also vital for hemodialysis patients [20].

$\mathbf{N}$-acetylcysteine: $\mathrm{N}$-acetylcysteine (NAC) is an active antioxidant that is safe in patients of hemodialysis and capable of scavenging free radicals [21]. A recently completed study shows that NAC effectively induces kidney function in patients with chronic hemodialysis. Studies have shown that NAC may also improve renal ischemia-reperfusion injury and seems to exacerbate vasodilation and vasodilatory effect. Vascular reactivity is associated with plasma nitric oxide (NO) and asymmetric dimethyl-L-arginine (ADMA) concentrations in dialysis patients. NAC has already been shown to decrease plasma levels of ADMA in patients with HD [22].

Folic acid and B12: Hemocysteine levels are high in hemodialysis patients. Decrease of its scavenging leads to risk factors for thrombosis and coronary artery disease, pulse pressure, arterial stiffness, and platelet aggregation $[23,24]$. Administration of folic acid and B12 inhibits the increase of homocysteine concentration [25].

L-carnitine: L-carnitine reduces the production of Acetyl-CoA, thereby reducing the production of free radicals, helping to repair oxidative stress to the phospholipid membrane, and reducing ischemia induced by apoptosis. L-carnitine is much higher in end-stage renal disease (ESRD) patients who do not receive hemodialysis than hemodialysis patients. L-carnitine reduces proinflammatory cytokines and improves protein synthesis and nitrogen balance in kidney disease patients or patients under hemodialysis or peritoneal dialysis [26].

Vitamins C, E: The imbalance between the anti-peroxide system and antioxidant system is comparably greater in patients with kidney failure. 
Dialysis also exacerbates oxidative stress to the extent that agents effective on oxidative stress are administered throughout hemodialysis. Oxidative stress is associated with decreased vitamin $C$ in dialysis patients $[4,27]$. Decreased antioxidants and increased oxidative products are among the factors contributing to activation of the red blood cell degradation pathway and erythropoietin resistance through red blood cell hemolysis, which is ultimately predisposing to renal anemia. Anemia is one of the most debilitating consequences of chronic kidney failure and may lead to fatal presentations. Therefore, studies have strongly recommended taking vitamin supplements such as $\mathrm{C}$ and $\mathrm{E}$ [28].

Nicotinamide: Control of Hyperphosphatemia is an important issue in hemodialysis patients. Nicotinamide has been shown to decrease the production of prognostic cytokines such as IL-1 $\beta$, IL-6, IL-8, and tumor necrosis. It is noteworthy that serum endotoxin activity is correlated with serum IL-6 levels in hemodialysis patients [29] and may be considered as a treatment of choice for reducing the endotoxin load in hemodialysis patients. Hyperphosphatemia treatment with nicotinamide also removes uremic toxins and phosphate, but outcomes of patient improvement have not yet been satisfactory [30].

Quercetin: Studies have shown that increased expression of FGF23 gene and parathyroid hormone leads to chronic kidney disease. In a rat model of adenine-induced chronic kidney disease, treatment with quercetin in hemodialysis patients improved renal function, reduced oxidative stress, and decreased serum fibroblast growth. Quercetin also decreases fibroblast growth factor (FGF23), kidney inflammation and kidney tubule damage. Further studies are needed to confirm these findings and evaluate the potential use of natural flavonoids, quercetin in the prevention and treatment of chronic renal disease [31].

Amino acids: Many proteins are lost. Inflammation and nitrogen catabolism and production are high in the hemodialysis process. In this case, the amino acid is helpful and healing.

Therefore, certain proteins such as amino acids, peptides, and oral amino acid supplements should increase throughout hemodialysis to improve albumin and total protein in hypoalbuminemic hemodialysis patients. This is also associated with CRP-related inflammation reduction and anemia improvement. Oral administration of amino acids can improve albumin and total protein in hypoalbuminemia in hemodialysis patients [32]. Phenols and flavonoids: Flavonoids are natural compounds that have pleiotropic properties, have potential therapeutic applications, and produce anti-inflammatory and anti-fibrotic effects. Polyphenols are substantially effective in heart disease, hemodialysis, vasodilatation, hypotension, and low triglyceride [33].

b-carotene: b-carotene are lipid-soluble molecules, whereas vitamin $\mathrm{C}$ resides in the aqueous phase.
During hemodialysis with increased oxidative stress increases malondialdehyde (MDA) and damage to cell membranes and DNA damages cells. Alpha tocopherol is an anthocyanin which neutralizes free radicals and prevents damage to cells [34].

Selenium: Although toxic in large doses, selenium is an essential trace element that functions as a cofactor for the reduction ofantioxidant enzymes such as glutathione peroxidase. The antioxidant benefits of selenium are purported to occur viaupregulation of the activity of this enzyme. Three small noncontrolled studies in a total of 40 HD patients investigated selenium therapy, with two finding decreased biomarkers of oxidative stress 84,85 and one finding no effect. 86 All three showed increased circulating selenium levels $[35,36]$.

Thea-lipoic acid: Thea-lipoic acid decreased plasma asymmetric dimethylarginine but had no effect on oxidized LDL, and coenzyme Q10 decreased plasma advanced oxidation end products but did not change plasma MDA. These two studies highlight the importance of the choice of the oxidative stress biomarker [37].

\section{Discussion}

The results show that elevated serum MDA concentration in kidney disease patients indicates increased oxidative stress in these patients, causing endothelial dysfunction, increase in acute phase inflammation and acceleration of development of heart disease in dialysis patients. Elevated serum MDA level is also the most common cause of death in these patients [38]. Kim et al. reported increased plasma levels of MDA and total antioxidant capacity in patients undergoing dialysis compared to the healthy group [39]. Anemia is caused by kidney failure due to decreased production by the bone marrow. Erythropoietin (EPO) is produced by the kidneys in response to anemia. Anemia in hemodialysis patients is normocytic normochromic. The reticulocyte count is low relative to the anemia severity, and erythroid bone marrow is hypoplastic [40]. Toxic metabolites, called uremic toxins, directly inhibit erythropoiesis or mitigate the impact of erythropoietin on bone marrow [40]. In one study, ascorbic acid was found to significantly increase transferrin saturation, which also indicates the ability of ascorbic acid to release storage iron into the circulating iron, which is subsequently used to improve anemia [41]. Antioxidants have positive effects on hemodialysis patients. Hemodialysis patients are more susceptible to oxidative stress. This disease reduces vasodilatation and increases oxidative stress and endothelial toxicity $[42,43]$. Weinstein also reported a low level of GSH in hemodialysis patients. Decreased glutathione in post-dialysis compared to pre-dialysis is likely due to increased oxidative stress following dialysis [44]. Post-dialysis calcium levels are higher than 
pre-dialysis. One of the reasons for increased post-dialysis calcium is weight loss and fluid loss in these patients. Aluminum gels and rocaltrol (intestinal phosphate transport) prevent the absorption of phosphate in the intestine and stop the cycle [45]. Dietary agents are also involved in these patients. Studies show that serum zinc levels are often lower than normal in uremic patients and hemodialysis patients or are in the normal range [46]. Even patients who do not undergo dialysis but have kidney problems,they have disorders of rare element metabolism, but these disorders are usually more severe in dialysis patients [44]. Increased serum antioxidant level following zinc administration is associated with a significant increase in serum SOD and glutathione levels. The antioxidant activity of zinc can be partly attributed to these two agents [48]. Another antioxidant, vitamin E, is a protective agent against oxidative stress and prevents the oxidation of lipoproteins. This pathway acts against the zinc antioxidant pathway [49]. Dialysis quality also has an impact on the life expectancy of kidney failure patients undergoing hemodialysis, so that the mortality and morbidity of these patients decrease with increasing dialysis quality [50]. Antioxidants prevent kidney failure and its progression by influencing effective factors on hemodialysis. A variety of herbal and non-herbal antioxidants can be used to treat disorders and diseases [50-61]. In terms of clinical applications, antioxidant products can be used to treat oxidative stress related to dialysis and are practical drugs, especially since oxidative stress caused by dialysis is also very common.

\section{Author contributions}

The study for review, search and data collection, and preparation of the manuscript was done by all of authors. The author read and approved the final manuscript.

\section{Conflict of interest}

The author declares that he has no conflict of interest.

\section{Funding/Support}

This research was financially supported by the author.

\section{REFERENCES}

[1] Kohen R, Chevion S, Schwartz R, Berry E. Evaluation of the total low molecular weight antioxidant activity of plasma in health and diseases: a new approach. Cellular Pharmacol 1996; 3: 355-60.
[2] Kosch M, Levers A, Fobker M, Barenbrock M, Schaefer $\mathrm{RM}$, Rahn $\mathrm{KH}$, et al. Dialysis filter type determines the acute effect of haemodialysis on endothelial function and oxidative stress. Nephrology Dialysis Transpl 2003; 18(7):1370-5

[3] Schettler V, Wieland E, Methe H, Schuff-Werner P, Müller GA. Oxidative stress during dialysis: effect on free radical scavenging enzyme (FRSE) activities and glutathione (GSH) concentration in granulocytes. Nephrology, dialysis, transplantation: official publication of the European Dialysis and Transplant Association-European Renal Association 1998; 13(10):2588-93.

[4] Fumeron C, Nguyen-Khoa T, Saltiel C, Kebede M, Buisson C, Drüeke TB, et al. Effects of oral vitamin C supplementation on oxidative stress and inflammation status in haemodialysis patients. Nephrology Dialysis Transplantation. 2005; 20(9):1874-9.

[5] Salamunić I, Juretić D, Ljutić D. Effect of different dialysis membranes on erythrocyte antioxidant enzyme levels and scavenger systems related to free hemoglobin in serum of hemodialysis patients. Clin Chem laboratory Med 2003; 41(7):904-7.

[6] Yang X, Hou F, Wu Q, Zhou H, Liu Z, Yang Y, et al. Increased levels of advanced oxidation protein products are associated with atherosclerosis in chronic kidney disease. Zhonghua Nei Ke Za Zhi. 2005; 5(6): 342-344.

[7] 7. Kronenberg F, Lingenhel A, Neyer U, Lhotta K, König P, Auinger M, et al. Prevalence of dyslipidemic risk factors in hemodialysis and CAPD patients. Kidney Int 2003; 63:S113-S6.

[8] Siems W, Quast S, Carluccio F, Wiswedel I, Hirsch D, Augustin W, et al. Oxidative stress in chronic renal failure as a cardiovascular risk factor. Clin Nephrol 2002; 58:S12-9.

[9] Denhaerynck K, Manhaeve D, Dobbels F, Garzoni D, Nolte C, De Geest S. Prevalence and consequences of nonadherence to hemodialysis regimens. American J Critical Care. 2007; 16(3):222-35.

[10] Takaki J, Nishi T, Shimoyama H, Inada T, Matsuyama N, Sasaki T, et al. Possible variances of blood urea nitrogen, serum potassium and phosphorus levels and interdialytic weight gain accounted for compliance of hemodialysis patients. J Psych Res2003;55(6):525-9.

[11] Lee S-h, Molassiotis A. Dietary and fluid compliance in Chinese hemodialysis patients. Int J nursing Studies 2002; 39(7):695-7.

[12] 12. López CM, Burrowes JD, Gizis F, Brommage D. Dietary adherence in Hispanic patients receiving hemodialysis. J Renal Nutr 2007; 17(2):138-47.

[13] Schneider MS, Friend R, Whitaker P, Wadhwa NK. Fluid noncompliance and symptomatology in end-stage renal disease: cognitive and emotional variables. Health Psychol1991; 10(3):209.

[14] Gilbert T, Helton C, White LL. Compliance among American Indian hemodialysis patients. J Renal Nutr 1994; 4(1):19-26.

[15] Schiavon R, De Fanti E, Giavarina D, Biasioli S, Cavalcanti 
G, Guidi G. Serum paraoxonase activity is decreased in uremic patients. Clinica Chimica Acta 1996; 247(1-2):71-80.

[16] Mitobe M, Yoshida T, Sugiura H, Shirota S, Tsuchiya K, Nihei H. Oxidative stress decreases klotho expression in a mouse kidney cell line. Nephron Exp Nephrol 2005; 101(2):e67-e74.

[17] Torres P-U, Prie D, Molina-Bletry V, Beck L, Silve C, Friedlander G. Klotho: an antiaging protein involved in mineral and vitamin D metabolism. Kidney Int 2007; 71(8):730-7.

[18] Hryszko T, Rydzewska-Rosolowska A, Gozdzikiewicz J, Brzósko S, Koc-Zorawska E, Zelazowska-Rutkowska B. Cholecalciferol supplementation reduces soluble Klotho concentration in hemodialysis patients. Pol Arch Med Wewn 2013; 123(6): 277.

[19] Chesney RW, Han X, Patters AB. Taurine and the renal system. J Biomed Sci 2010; 17(1):S4.

[20] Okuno S, Ishimura E, Norimine K, Tsuboniwa N, Kagitani $\mathrm{S}$, Yamakawa $\mathrm{K}$, et al. Serum adiponectin and bone mineral density in male hemodialysis patients. Osteoporosis Int 2012; 23(7):2027-35.

[21] Tepel M, Van Der Giet M, Statz M, Jankowski J, Zidek W. The antioxidant acetylcysteine reduces cardiovascular events in patients with end-stage renal failure: a randomized, controlled trial. Circulation. 2003; 107(7):992-5.

[22] Thaha M, Pranawa W, Yogiantoro M, Tomino Y. Intravenous $\mathrm{N}$-acetylcysteine during hemodialysis reduces asymmetric dimethylarginine level in end-stage renal disease patients. Clin Nephrol 2008; 69(1): 32-34.

[23] Steenge GR, Verhoef P, Katan MB. Betaine supplementation lowers plasma homocysteine in healthy men and women. J Nutr 2003; 133(5):1291-5.

[24] Tsai J-C, Kuo H-T, Chiu Y-W, Hwang S-J, Chuang H-Y, Chang J-M, et al. Correlation of plasma homocysteine level with arterial stiffness and pulse pressure in hemodialysis patients. Atherosclerosis. 2005; 182(1):121-7.

[25] Sohrvardi S, Azmandian J, Daryaee F, Mohammadpoor A. Plasma Homocysteine Concentration in Hemodialysis Patients of Kerman/Iran in 2005. J Kerman Uni Med Sci 2014(2): 23 .

[26] Mortazavi M, Seyrafian S, Taheri S, Eshaghian A, Ghesami M. Effect of L-Carnitine on Serum Albumin Level of Hemodialysis Patients; A Placebo Controlled Randomized Clinical Trial. - Isfahan Med School 2011; 28(119): 34-5.

[27] Nguyen-Khoa T, Massy ZA, De Bandt JP, Kebede M, Salama L, Lambrey G, et al. Oxidative stress and haemodialysis: role of inflammation and duration of dialysis treatment. Nephrol Dialysis Transpl 2001; $16(2): 335-40$

[28] Attallah N, Osman-Malik Y, Frinak S, Besarab A. Effect of intravenous ascorbic acid in hemodialysis patients with EPO-hyporesponsive anemia and hyperferritinemia. American J Kidney Dis 2006; 47(4): 644-54.

[29] Bossola M, Di Stasio E, Sanguinetti M, Posteraro B,
Antocicco M, Pepe G, et al. Serum endotoxin activity measured with endotoxin activity assay is associated with serum interleukin-6 levels in patients on chronic hemodialysis. Blood Purification. 2016;44: 294-300..

[30] Lenglet A, Fabresse N, Taupin M, Gomila C, Liabeuf S, Kamel S, et al. Does the Administration of Sevelamer or Nicotinamide Modify Uremic Toxins or Endotoxemia in Chronic Hemodialysis Patients? Drugs 2019; 79(8):855-62.

[31] Yang H, Song Y, Liang Y-n, Li R. Quercetin treatment improves renal function and protects the kidney in a rat model of adenine-induced chronic kidney disease. Medical science monitor: Int Med J experimental Clin Res 2018; 24:4760.

[32] Bolasco P, Caria S, Cupisti A, Secci R, Saverio Dioguardi F. A novel amino acids oral supplementation in hemodialysis patients: a pilot study. Renal failure. 2011; $33(1): 1-5$

[33] Vogel G, Tuchweber B, Trost W, Mengs U. Protection by silibinin against Amanita phalloides intoxication in beagles. Toxicol Applied Pharmacol1984; 73(3):355-62.

[34] Ergola PE, Krauth M, Huff JWet al.Effect of bardoxolone methyl onkidney function in patients with T2D and Stage 3b-4 CKD.Am J Nephrol 2011; 33: 469-476.

[35] Damowicz A, Trafikowska U, Trafikowska Aet al.Effect oferythropoietin therapy and selenium supplementation on selectedantioxidant parameters in blood of uremic patients on long-termhemodialysis.Med Sci Monit 2002;8: CR202CR205.

[36] Ichard MJ, Ducros V, Foret Met al.Reversal of selenium and zincdeficiencies in chronic hemodialysis patients by intravenous sodiumselenite and zinc gluconate supplementation. Time -course ofglutathione peroxidase repletion and lipid peroxidation decrease.BiolTrace Elem Res1993; 39: 149-159.

[37] Himmelfarb J, Phinney S, Ikizler TAet al.Gamma-tocopherol anddocosahexaenoic acid decrease inflammation in dialysis patients.J Ren Nutr 2007;17: 296304 .

[38] Trimarchi H, Mongitore M, Baglioni P, Forrester M, Freixas E, Schropp M, et al. N-acetylcysteine reduces malondialdehyde levels in chronic hemodialysis patients--a pilot study. Clin Nephrol 2003; 59(6):441-6.

[39] Kim SB, Yang WS, Min WK, Lee SK, Park JS. Reduced oxidative stress in hypoalbuminemic CAPD patients. Peritoneal Dialysis Int 2000; 20(3):290-4.

[40] Rahimian SH, The effect of intravenous ascorbic acid on correction of anemia in hemodialysis patients with renal failure. J Shahid Sadoughi Uni Med Sci 2006; 14(1): 3-8.

[41] Viganò G, Benigni A, Mendogni D, Mingardi G, Mecca G, Remuzzi G. Recombinant human erythropoietin to correct uremic bleeding. American J Kidney Dis 1991;18(1):44-9.

[42] Behradmanesh S, Nasri P. Serum cholesterol and LDL-C in association with level of diastolic blood pressure in type 2 diabetic patients. J Renal Injury Prev 2012; 1(1): 23.

[43] Ziouzenkova O, Asatryan L, Tetta C, Wratten ML, Hwang J, Sevanian A. Oxidative stress during ex vivo 
hemodialysis of blood is decreased by a novel hemolipodialysis procedure utilizing antioxidants. Free Radical Biology and Med 2002; 33(2): 248-58.

[44] Weinstein T CA, Korzets A,Boaz M, Ori Y,Herman M,et al. Evidence for impaire defenced mechanisms against oxidative stress. . Nephrol Dial Transplant. 2000; 15(2): $883-7$.

[45] Moe SM, Drüeke TB. Management of secondary hyperparathyroidism: the importance and the challenge of controlling parathyroid hormone levels without elevating calcium, phosphorus, and calcium-phosphorus product. American J Nephrol 2003; 23(6):369-79.

[46] Lee SH, Huang JW, Hung KY, Leu LJ, Kan YT, Yang CS, et al. Trace Metals' abnormalities in hemodialysis patients: relationship with medications. Artificial Organs. 2000; 24(11):841-4

[47] Lin T, Chen J, Liaw J, Juang J. Trace elements and lipid peroxidation in uremic patients on hemodialysis. Biological Trace Element Res. 1996; 51(3):277-83.

[48] Alscher DM, Braun N, Biegger D, Stuelten C, Gawronski K, Mürdter TE, et al. Induction of metallothionein in proximal tubular cells by zinc and its potential as an endogenous antioxidant. Kidney Blood Pressure Res 2005; 28(3):127-33.

[49] Bunk M, Dnistrian A, Schwartz M, Rivlin R. Dietary zinc deficiency decreases plasma concentrations of vitamin $\mathrm{E}$. Proceedings of the Society for Experimental Biol Med 1989; 190(4):379-84.

[50] Solati M, Raiezadeh F, Pezeshk P. Impact of hemodialysis on serum paraoxonase activity and total antioxidant capacity in patients with end-stage renal disease. Res Med 2003; 27(4):265-71.

[51] Moayeri A, Azimi M, Karimi E, Aidy A, Abbasi N. Attenuation of Morphine Withdrawal Syndrome by Prosopis Farcta Extract and Its Bioactive Component Luteolin in Comparison with Clonidine in Rats. Med Sci Monitor Basic Res 2018; 24(9): 151-158.

[52] Abbaszadeh S, Karami N, Bahmani F, Abbasi N, Atefi E.
Headache and herbal medicine: An ethnobotanical study of Shahrekord, Southwest of Iran. Plant Biotechnol Persa. 2019; 1 (1): 4-9.

[53] Abbasi N, Khosravi A, Aidy A, Shafiei M. Biphasic response to luteolin in MG-63 osteoblast-like cells under high glucose-induced oxidative stress. Iranian J Med Sci 2016; 41(2): 118-125.

[54] Beiranvand F, Alizadeh M. Plants for remedies of diabetes mellitus in Iran. Plant Biotechnol Persa. 2019; 1 (1): 36-38.

[55] Karimi E. Alzheimer's: Phytotherapy and the most important herbs in the treatment of Alzheimer's. Plant Biotechnol Persa. 2020; 2 (1): 61-62.

[56] Valadi A, Nasri S, Abbasi N, Amin GR. Antinociceptive and anti-inflammatory effects of hydroalchoholic extract of Anethum graveolens L. seed. J Med Plants 2010; 9(34):124-130.

[57] Karami N, Karimi M, Bahmani M. Constipation: An ethno-botanical study of medicinal plants used for constipation in Shahrekord city, Chaharmahal \& Bakhtiari province, Iran. Plant Biotechnol Persa. 2020; 2 (1): 1-7.

[58] Bahmani M, Mozaffari Nejad AS, Shah NA, Shah SA, Rafieian-Kopaei M, Mahmoodnia L. Survey on ethnobotanical uses of anti-cancer herbs in southern region of Ilam, west Iran. J Biolog Res 2017; 90(1): 5939.

[59] Abbaszadeh S, Andevari AN, Koohpayeh A, Naghdi N, Alizadeh M, Beyranvand F, Harsej Z. Folklore medicinal plants used in liver disease: A review. Int $\mathrm{J}$ Green Pharmacy 2018; 12(3): 463-472.

[60] Sedighi M, Sewell R.D.E, Nazari A, Abbaszadeh S, Cheraghi M, Amini A, Heydari Z, Rafieian-Kopaei M. A review on the most important medicinal plants effective in cardiac ischemia-reperfusion injury. Current Pharmaceutical Design 2019; 25(3): 352-358.

[61] Nouri A, Heidarian E, Amini-Khoei H, Abbaszadeh S, Basati G. Quercetin through mitigation of inflammatory response and oxidative stress exerts protective effects in rat model of diclofenac-induced liver toxicity. J Pharmacy Pharmacog Res 7(3): 2019, Pages 200-212. 